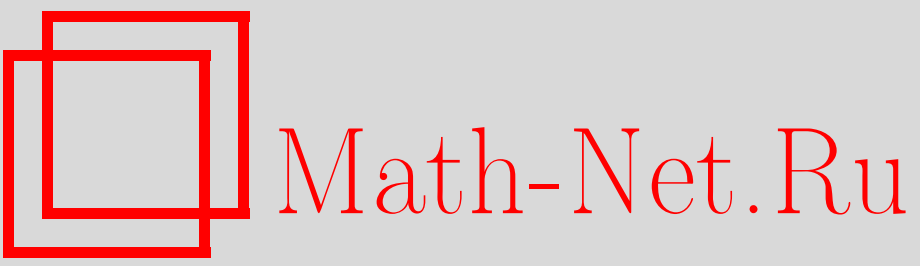

А. В. Михайлов, В. В. Соколов, Интегрируемые обыкновенные дифференциальные уравнения на свободных ассоциативных алгебрах, ТМФ, 2000, том 122 , номер 1, 88-101

DOI: https://doi.org/10.4213/tmf557

Использование Общероссийского математического портала Math-Net.Ru подразумевает, что вы прочитали и согласны с пользовательским соглашением

http://www . mathnet.ru/rus/agreement

Параметры загрузки:

IP : 35.173 .137 .237

26 апреля 2023 г., 18:19:01 


\section{ИНТЕГРИРУЕМЫЕ ОБЫКНОВЕННЫЕ ДИФФЕРЕНЦИАЛЬНЫЕ УРАВНЕНИЯ НА СВОБОДНЫХ АССОЦИАТИВНЫХ АЛГЕБРАХ}

Рассматривается задача классификации интегрируемых обыкновенных нелинейных дифференциальных уравнений с зависимой переменной, принадлежащей свободной ассоциативной алгебре $\mathcal{M}$. Каждое такое уравнение допускает интегрируемую $(m \times m)$-матричную редукцию для любого $m$. В качестве критерия интегрируемости используется существование симметрий и/или первых интегралов, принадлежащих алгебре $\mathcal{M}$.

\section{1. ВВЕДЕНИЕ}

Для уравнений в частных производных один из наиболее эффективных способов выделения точно интегрируемых случаев связан с сушествованием высших симметрий и законов сохранения [1-3]. Наша цель - обобшить этот подход на случай обыкновенных дифференциальных уравнений вида

$$
M_{t}=F(M, C),
$$

где $M(t)$ и постоянный элемент $C$ принадлежат свободной ассоциативной алгебре $\mathcal{M}$, а $F$ - некоторый (некоммутативный) многочлен. Аналогичная задача для уравнений в частных производных на свободных ассоциативных алгебрах, существенно более близкая к стандартной теории, рассматривалась в работах $[4,5]$. В работе [6] была сделана попытка обобщения теста Пенлеве на случай уравнений на ассоциативных алгебрах.

Задача нахождения интегрируемых случаев для уравнений на свободных ассоциативных алгебрах важна потому, что каждое интегрируемое уравнение допускает множество различных интегрируемых конечномерных редукций. Например, можно считать $M$ и $C$ матрицами произвольной размерности. В соответствуюшем интегрируемом матричном уравнении возможны дальнейшие редукции, связанные со спецификацией вида матриц $M$ и $C$. В частности, таким образом из интегрируемого уравнения

*Applied Math. Department, University of Leeds, Leeds, UK; Институт теоретической физики им. Л. Д. Ландау РАН, Москва, Россия

${ }^{\dagger}$ Центр нелинейных исследований при Институте теоретической физики им. Л.Д. Ландау РАН, Москва, Россия 
$M_{t}=\left[M^{2}, C\right]$ на $\mathcal{M}$ могут быть получены неабелевы цепочки Вольтерра. Отдельной интересной задачей является изучение редукций уравнения (1), приводящих к классическим волчкам.

В данной работе мы обобщаем такие основные понятия симметрийного подхода, как инфинитезимальная симметрия, первый интеграл, рекурсионный и гамильтонов операторы и др. для случая уравнений на алгебре $\mathcal{M}$. Все наши определения близки к стандартным (по поводу которых мы отсылаем читателя к широко известной книге Олвера [7]) и легко обобшаются на случай ассоциативных алгебр с любым конечным числом образующих.

Кроме того, мы приводим ответы для простейших задач классификации интегрируемых уравнений на алгебре $\mathcal{M}$ и список локальных гамильтоновых операторов малых порядков.

\section{2. ПЕРВЫЕ ИНТЕГРАЛЫ И СИММЕТРИИ ДЛЯ УРАВНЕНИЙ НА СВОБОДНЫХ АССОЦИАТИВНЫХ АЛГЕБРАХ}

Обозначим через $\mathcal{M}$ свободную ассоциативную алгебру над полем $\mathbb{C}$ (или $\mathbb{R}$ ) с единищей $I d$ и образуюшими $M$ и $C$. Все основные понятия, такие как симметрия, первый интеграл, рекурсионный и гамильтонов операторы и др., будут определены исключительно в терминах заданных на $\mathcal{M}$ операций умножения $\circ$, сложения и умножения на константы (которые мы будем обозначать буквами $\alpha, \beta, \gamma, \ldots$ ). В качестве примера будет использовано уравнение

$$
M_{t}=M^{2} \circ C-C \circ M^{2} .
$$

Это уравнение обладает представлением Лакса, но мы намеренно не будем учитывать это обстоятельство, поскольку поиск лаксовой пары не является алгоритмической процедурой и для других уравнений пара может не быть известна.

ОПРЕДЕЛЕНИЕ 1. Назовем $G \in \mathcal{M}$ генератором инфинитезимальной симметрии (или просто симметрией) для уравнения (1), если потоки $M_{\tau}=G$ и $M_{t}=F$ коммутируют, т.е. если производная $d G / d t$, вычисленная в силу уравнения (1), и производная $d F / d \tau$, вычисленная в силу уравнения $M_{\tau}=G$, дают один и тот же элемент $\mathcal{M}$.

Что касается первых интегралов, то вместо того, чтобы использовать понятие следа, которьй не определен на алгебре $\mathcal{M}$, мы введем следуюшее отношение эквивалентности.

ОПРЕДЕЛЕнИЕ 2. Два элемента $f_{1}$ и $f_{2}$ из $\mathcal{M}$ назовем эквивалентными $\left(f_{1} \sim f_{2}\right)$, если $f_{1}$ может быть получен из $f_{2}$ с помошью циклических перестановок в его мономах.

Например, $M \circ C \circ M \sim C \circ M \circ M$, и, конечно, всякий коммутатор эквивалентен нулю.

ОПРЕДЕЛЕнИЕ 3. Элемент $h$ алгебры $\mathcal{M}$ назовем первым интегралом уравнения (1), если $d h / d t \sim 0$. Первые интегралы $h_{1}$ и $h_{2}$ называются эквивалентными, если $h_{1}-h_{2} \sim 0$. 
Это определение напоминает определение плотности локального закона сохранения для эволюционных уравнений в частных производных [7]. И первые интегралы, и плотности фактически определяются как классы эквивалентности. Разница заключается только в выборе отношения эквивалентости.

Согласно нашему определению элементы $R_{n}=M^{n}$ являются нетривиальными (т.е. не эквивалентными нулю) первыми интегралами уравнения (2). Действительно,

$$
\frac{d}{d t} M^{n}=\sum_{k=1}^{n} M^{k-1} \circ\left(M^{2} \circ C-C \circ M^{2}\right) \circ M^{n-k},
$$

и, как легко видеть, каждое слагаемое в этой сумме эквивалентно нулю.

Правая часть уравнения (2) является многочленом, однородным относительно растяжений $C \rightarrow \mu C$ и $M \rightarrow \nu M$. Для многочленов такого типа показатели степеней $\mu$ и $\nu$ мы будем называть весами. На всей алгебре $\mathcal{M}$ определена двойная градуировка $\mathcal{M}=\bigoplus \mathcal{M}_{n m}$, где $\mathcal{M}_{n m}$ состоит из многочленов, имеющих вес $n$ по $C$ и вес $m$ по $M$.

Следуюшее утверждение фактически очевидно.

ЛЕмма 1. Если $I$ и $G$ являются соответственно первым интегралом и симметрией для уравнения, правая часть которого - многочлен двойной однородности, то любая однородная компонента многочленов $I$ u $G\left(\right.$ m.е. проекиия на $\left.\mathcal{M}_{n т}\right)$ также является первым интегралом и соответственно симметрией.

Непосредственное вычисление методом неопределенных коэффициентов однородных первых интегралов малых весов для уравнения (2) является простым упражнением. При этом дело сводится к решению однородной линейной переопределенной системы для коэффициентов. Например, всякий однородный многочлен веса $(2,2)$ эквивалентен многочлену вида

$$
P_{2,2}=\alpha C^{2} \circ M^{2}+\beta C \circ M \circ C \circ M .
$$

Легко проверить, что $d P_{2,2} / d t \sim 0$, если и только если $\alpha=2 \beta$. Следовательно, величина

$$
I_{2,2}=2 C^{2} \circ M^{2}+C \circ M \circ C \circ M
$$

есть первый интеграл для уравнения (2). Аналогично можно найти первые интегралы

$$
I_{3,2}=C^{3} \circ M^{2}+C^{2} \circ M \circ C \circ M, \quad I_{2,3}=C^{2} \circ M^{3}+C \circ M \circ C \circ M^{2} .
$$

Напомним, что интегралами уравнения (2) являются $I_{0, n}=M^{n}$. Более того, можно доказать, что $I_{n}=(M+C)^{n}$ - интеграл для любого натурального $n$. Все приведенные выше интегралы эквивалентны некоторым однородным компонентам интегралов $I_{j}$.

Аналогичные вычисления позволяют найти однородные симметрии $S_{n, m}$ для уравнения (2) при небольших $n$ и $m$. Например, чтобы найти симметрию $S_{2,2}$, мы начинаем с обшего анзаца

$$
\begin{aligned}
Q_{2,2}= & \alpha_{1} C^{2} \circ M^{2}+\alpha_{2} C \circ M \circ C \circ M+\alpha_{3} C \circ M^{2} \circ C+ \\
& +\alpha_{4} M \circ C^{2} \circ M+\alpha_{5} M \circ C \circ M \circ C+\alpha_{6} M^{2} \circ C^{2} .
\end{aligned}
$$

Нетрудно проверить, что $Q_{2,2}$ будет симметрией для уравнения (2) тогда и только тогда, когда $\alpha_{3}=\alpha_{4}=0$ и $\alpha_{1}=\alpha_{2}=-\alpha_{5}=-\alpha_{6}$. 


\section{3. ПРИМЕРЫ ИНТЕГРИРУЕМЫХ УРАВНЕНИЙ НА $\mathcal{M}$}

Уравнение (2) обладает бесконечной серией первых интегралов и симметрий, что в принципе позволяет проинтегрировать его конечномерные матричные редукции. Отметим, что в случае, когда $M$ - кососимметрическая $(3 \times 3)$-матрица, а матрица $C$ диагональна, уравнение (2) совпадает со знаменитым волчком Эйлера.

Возникает естественньй вопрос: существуют ли другие уравнения на $\mathcal{M}$, обладаюшие симметриями и/или первыми интегралами?

Произвольное уравнение однородности $(1,2)$ на $\mathcal{M}$ имеет вид

$$
M_{t}=\alpha M^{2} \circ C+\beta M \circ C \circ M+\gamma C \circ M^{2} .
$$

Попытаемся выяснить, при каких соотношениях между константами уравнение (5) имеет симметрии. Согласно лемме 1 мы можем независимо изучать наличие симметрий в каждой однородной компоненте.

Уравнение (5) всегда имеет очевидную последовательность симметрий $S_{1, n}=$ $M C^{n}-C^{n} M, n=1,2, \ldots$ В матричном случае такие симметрии соответствовали бы инвариантности уравнения относительно сопряжений матрицами $C^{n}$. Симметрии $S_{1, n}$ мы назовем тривиальными и будем опускать во всех последующих формулах.

Мы исследовали уравнение (5) на предмет сушествования однородных симметрий $S_{n, m} \in \mathcal{M}_{n, m}$ при $n+m \leqslant 10$ с помошью компьютера. Легко видеть, что для однородного многочлена $P_{n, m} \in \mathcal{M}_{n, m}$ обшего вида определение симметрии приводит к переопределенной системе квадратичных уравнений относительно коэффициентов $P_{n, m}$ и параметров $\alpha, \beta, \gamma$ в уравнении (5).

ПРЕДЛОЖЕНИЕ 1. С точностью до растяжения $t$ каждое уравнение вида (5), обладающее нетривиальной симметрией $S_{n, m} \in \mathcal{M}, n+m \leqslant 10$, совпадает с одним из следующих уравнений:

$$
\begin{aligned}
& M_{t}=\left[M^{2}, C\right], \\
& M_{t}=[M, M \circ C], \\
& M_{t}=[C \circ M, M], \\
& M_{t}=M \circ C \circ M .
\end{aligned}
$$

Хотя в принципе не исключено существование уравнения, у которого нет симметрий $S_{n, m}$ с $n+m \leqslant 10$, но есть симметрии более высоких степеней, практически это выглядит весьма маловероятным.

С первым уравнением списка (6) мы уже знакомы. Уравнения (7) и (8) эквивалентны

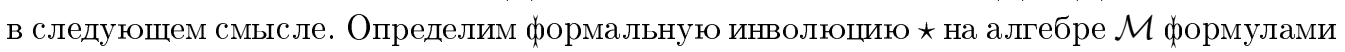

$$
\begin{gathered}
M^{\star}=M, \quad C^{\star}=C, \quad(A \circ B)^{\star}=B^{\star} \circ A^{\star}, \\
(\alpha A+\beta B)^{\star}=\alpha A^{\star}+\beta B^{\star}, \quad A, B \in \mathcal{M} .
\end{gathered}
$$


Если уравнение $M_{t}=F$ имеет симметрию $M_{\tau}=G$, то очевидно, что $M_{\tau}=G^{\star}$ - симметрия для уравнения $M_{t}=F^{\star}$. Уравнение (8) является результатом применения инволюции $\star$ к (7). Поэтому все результаты, касаюшиеся (7), автоматически переносятся на уравнение (8).

Явный вид нескольких первых симметрий для уравнений (7), (9) привел нас к следующему утверждению, которое доказывается прямым вычислением.

ПРЕДЛОЖЕНИЕ 2. Уравнение (7) имеет коммутирующие друг с другом симметрии

$$
M_{\tau_{n, m}}=\left[M, M^{n-1} \circ C^{m}\right], \quad n, m \in \mathbb{N} .
$$

Уравнение (9) имеет коммутирующие симметрии

$$
M_{\tau_{2, m}}=M \circ C^{m} \circ M, \quad m \in \mathbb{N} .
$$

Отметим, что наличие бесконечных иерархий симметрий существенно отличает интегрируемые уравнения (1) от систем обыкновенных дифференциальный уравнений и, наоборот, приближает их по свойствам к интегрируемым уравнениям в частных производных.

Описание уравнений (5), обладаюших первыми интегралами $I_{n, m}$, является еще одной классификационной задачей, которая при малых $n, m$ может быть решена на компютере.

ПРЕДЛОЖЕНИЕ 3. Уравнение (5) обладает первыми интегралами вида $I_{n, 0}=M^{n}$ тогда и только тогда, когда $\alpha+\beta+\gamma=0$. Если уравнение (5) имеет хотя бъ один интеграл $I_{n, m}$, где $n>0, m>0$ u $n+m \leqslant 10$, то с точностью до растяжения $t$ оно совпадает с уравнением (6).

В частности, уравнение (9) совсем не имеет первых интегралов, а уравнение (7) обладает только очевидными орбитными интегралами $I_{n, 0}$. Однако у обоих уравнений есть бесконечные иерархии симметрий. Это указывает на их возможную " $C$-интегрируемость" (подобный эффект для уравнений в частных производных отмечался в [1]).

И на самом деле, если $M, C$ являются $(n \times n)$-матрицами, для уравнения $(9)$ нетрудно написать явную формулу [8] для решения задачи Коши $M(0)=M_{0}$ :

$$
M(t)=\left(I d-M_{0} C t\right)^{-1} M_{0} .
$$

Уравнение (7) в матричном случае может быть проинтегрировано следующим образом. Пусть $B$ - произвольная постоянная матрица и $T$ - обшее решение линейного дифференциального уравнения с постоянными коэффициентами

$$
T_{t}=B T C
$$

Тогда $M(t)=T^{-1} B T$ удовлетворяет уравнению (7). Более того, если $T\left(t, \tau_{n, m}\right)$ - общее решение уравнения (13) и $T_{\tau_{n, m}}=B^{n} T C^{m}$ (очевидно, что эти уравнения совместны), то $M\left(t, \tau_{n, m}\right)=T^{-1} B T$ удовлетворяет одновременно уравнениям (7) и (11). 
Уравнения (6) и (7) обладают кубическими по $M$ симметриями. Возникает следующий естественный вопрос: сушествуют ли отличные от этих симметрий уравнения вида

$$
M_{t}=a M^{3} \circ C+b M^{2} \circ C \circ M+c M \circ C \circ M^{2}+d C \circ M^{3},
$$

обладаюшие симметриями и/или первыми интегралами?

Ответ формулируется следующим образом.

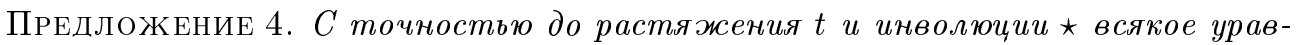
нение вида (14), имеющее нетривиальную симметрию $S_{n, m} \in \mathcal{M}, \quad n+m \leqslant 10$, совпадает с одним из следующих:

$$
\begin{aligned}
& M_{t}=\left[M^{3}, C\right], \\
& M_{t}=\left[M, C \circ M^{2}\right], \\
& M_{t}=[M \circ C \circ M, M], \\
& M_{t}=M^{3} \circ C-M^{2} \circ C \circ M+M \circ C \circ M^{2}-C \circ M^{3} .
\end{aligned}
$$

Два первых уравнения (15) и (16) являются соответственно симметриями уравнений $(6)$ и (7).

Уравнение (18) имеет однородные симметрии $S_{1,2 k-1}$, но симметрии однородности $S_{1,2 k}$ у него отсутствуют. Можно проверить, что если $M$ удовлетворяет уравнению (18), то элемент $N=M^{2}$ является решением уравнения (6), т.е. $N_{t}=\left[N^{2}, C\right]$. В скалярном случае мы считали бы такие уравнения точечно-эквивалентными. Для матричных уравнений, если мы знаем некоторое решение (18), то знаем и решение (6), но не наоборот.

Обобшая (18), можно найти уравнение для "корня $n$-й степени" из $N$. А именно легко показать, что если $M$ удовлетворяет уравнению

$$
M_{t}=M^{n+1} \circ C-M^{n} \circ C \circ M+M \circ C \circ M^{n}-C \circ M^{n+1},
$$

то $N=M^{n}$ является решением уравнения (6). Уравнение (19) обладает симметриями $S_{1, k n+1}, k=0,1, \ldots$, но у него нет симметрий с однородностями $(1, p)$, где $p \not \equiv 1$ $(\bmod n)$.

Уравнение (17) является наиболее интересным из приведенного выше списка. Оно обладает симметриями веса $(1, n)$ для любого $n$. Формально это уравнение может быть следуюшим образом связано с расширением иерархии симметрий уравнения (6). Если пополнить алгебру $\mathcal{M}$ обратным элементом $N=M^{-1}$, то согласно (17) имеем $N_{t}=$ $\left[N^{-1}, C\right]$. Последнее уравнение является неполиномиальной симметрией уравнения (6). Вообще говоря, для матричных редукций обратный элемент может не существовать (как, например, в случае, когда $(3 \times 3)$-матрица $M$ кососимметрична) и тогда $(17)$ никак не связано с иерархией уравнения (6).

Можно проверить, что уравнение

$$
M_{t}=\left[M, M^{n} \circ C \circ M^{n}\right]
$$

связано с (17) подстановкой $N=M^{n}$. Подобно (19) это уравнение имеет лакуны длины $n$ в иерархии симметрий. 


\section{4. ГАМИЛЬТОНОВЫ И РЕКУРСИОННЫЕ ОПЕРАТОРЫ НА АЛГЕБРЕ $\mathcal{M}$}

4.1. Основные определения. В этом разделе мы даем определения таких понятий, как производная Фреше, вариационная производная, гамильтонов оператор и др., годящиеся для уравнений на свободной алгебре $\mathcal{M}$. По-прежнему мы будем использовать уравнение (2) для иллюстрации наших определений.

Для всякого элемента $a \in \mathcal{M}$ мы определим операторы левого $\left(L_{a}\right)$ и правого $\left(R_{a}\right)$ умножения, которые отображают $\mathcal{M}$ в себя по следующему правилу:

$$
L_{a}(b)=a \circ b, \quad R_{a}(b)=b \circ a,
$$

для любого $b \in \mathcal{M}$. Ясно, что операторы $L_{a}$ и $R_{a}$ линейны по $a$. Из ассоциативности алгебры $\mathcal{M}$ следует, что для любых $a$ и $b$ выполнены тождества $R_{a} L_{b}=L_{b} R_{a}$ и

$$
L_{a \circ b}=L_{a} L_{b}, \quad R_{a \circ b}=R_{b} R_{a}
$$

Обозначим через $\mathcal{O}$ алгебру, порожденную генераторами $L_{M}, L_{C}, R_{M}, R_{C}$ и соотношениями

$$
R_{M} L_{M}=L_{M} R_{M}, \quad R_{M} L_{C}=L_{C} R_{M}, \quad R_{C} L_{M}=L_{M} R_{C}, \quad R_{C} L_{C}=L_{C} R_{C}
$$

Будем называть $\mathcal{O}$ алгеброй локальных операторов на $\mathcal{M}$. В соответствии с $(21)$ операторы умножения на любой элемент $\mathcal{M}$ являются элементами $\mathcal{O}$. Обозначим через $i d$ единичный элемент $\mathcal{O}$, т.е. оператор умножения на $I d$.

Градуировка на $\mathcal{M}$ индуцирует градуировку $\mathcal{O}=\bigoplus \mathcal{O}_{n, m}$ так, что если $A \in \mathcal{O}_{n, m}$, то $A: \mathcal{M}_{p, q} \rightarrow \mathcal{M}_{p+n, q+m}$. Элементы $\mathcal{O}_{n, m}$ будем называть однородными операторами веса $(n, m)$.

Согласно следуюшему определению производная $\Phi$ реше $a_{*}$ от любого элемента $a \in \mathcal{M}$ принадлежит $\mathcal{O}$.

ОПРЕДЕЛЕНИЕ 4 . Пусть $a=a(M, C)$ - любой элемент $\mathcal{M}$. Тогда производная $\Phi$ реше $a_{*} \in \mathcal{O}$ от $a$ единственным образом определяется формулой

$$
\left.\frac{d}{d \epsilon} a(M+\epsilon \delta M, C)\right|_{\epsilon=0}=a_{*}(\delta M)
$$

Например, следуя определению, вычислим производную $Ф$ реше $F_{*}$ от правой части $F(M, C)=M^{2} \circ C-C \circ M^{2}$ уравнения (2):

$$
\begin{aligned}
& \left.\frac{d}{d \epsilon}(F(M+\epsilon \delta M, C))\right|_{\epsilon=0}=\left.\frac{d}{d \epsilon}\left((M+\epsilon \delta M)^{2} \circ C-C \circ(M+\epsilon \delta M)^{2}\right)\right|_{\epsilon=0}= \\
& \quad=\delta M \circ M \circ C+M \circ \delta M \circ C-C \circ \delta M \circ M-C \circ M \circ \delta M=F_{*}(\delta M) .
\end{aligned}
$$

Следовательно,

$$
F_{*}=R_{C} R_{M}+L_{M} R_{C}-L_{C} R_{M}-L_{C} L_{M}
$$


Если $F$ и $G$ являются правыми частями двух эволюционных уравнений на $\mathcal{M}$, то правая часть $K \in \mathcal{M}$ коммутатора потоков, соответствуюших $F$ и $G$, определяется формулой

$$
K=G_{*}(F)-F_{*}(G) .
$$

Как обычно [7], можно переформулировать определение симметрии в терминах производных Фреше. Скажем, что $G$ является генератором симметрии для уравнения $M_{t}=F$, если

$$
G_{*}(F)=F_{*}(G)
$$

Соотношение (23) называется определяющим уравнением для симметрий.

Дифференцирование в силу уравнения $M_{t}=F$ продолжается на алгебру $\mathcal{O}$ формулой

$$
\frac{d}{d t} L_{a}=L_{a_{\star}(F)}, \quad \frac{d}{d t} R_{a}=R_{a_{\star}(F)},
$$

где $a-$ произвольный элемент $\mathcal{M}$.

Формальная инволюция $\star$ индуцирует инволюцию на $\mathcal{O}$ :

$$
L_{a}^{\star}=R_{a}, \quad R_{a}^{\star}=L_{a} .
$$

В частности, $R_{M}^{\star}=L_{M}, L_{C}^{\star}=R_{C}$. Полезно помнить, что для любых $a, b \in \mathcal{M}$ и $Q \in \mathcal{M}$

$$
a \circ Q(b) \sim b \circ Q^{\star}(a) .
$$

Оператор $Q \in \mathcal{O}$ называется симметричным (кососимметричным), если $Q^{\star}=Q$ $\left(Q^{\star}=-Q\right)$. Например, оператор $\operatorname{ad}_{M}=L_{M}-R_{M}$ кососимметричен.

4.2. Гамильтоновы структуры на $\mathcal{M}$. Обшее гамильтоново уравнение на $\mathcal{M}$ имеет вид

$$
M_{t}=\Theta\left(\operatorname{grad}_{M}(H(M, C))\right),
$$

где $H(M, C) \in \mathcal{M}$ - гамильтониан уравнения, $\Theta$ - гамильтонов оператор. Ниже мы изучаем локальные гамильтоновы операторы, т.е. предполагаем, что $\Theta \in \mathcal{O}$.

Градиент $\operatorname{grad}_{M}(a)$ от $a \in \mathcal{M}$ снова элемент $\mathcal{M}$, который определяется следующим образом.

ОПРЕДЕЛЕНИЕ 5. Пусть $a(M, C) \in \mathcal{M}$. Тогда градиент $\operatorname{grad}_{M}(a(M, C))$ единственным образом определяется из условия

$$
\left.\frac{d}{d \epsilon} a(M+\epsilon \delta M, C)\right|_{\epsilon=0} \sim \delta M \circ \operatorname{grad}_{M}(a(M, C))
$$

Легко проверить, что если $H_{1} \sim H_{2}, H_{i} \in \mathcal{M}$, $\operatorname{Tograd}_{M}\left(H_{1}\right)=\operatorname{grad}_{M}\left(H_{2}\right)$, и, в частности, если $H_{1} \sim 0$, то $\operatorname{grad}_{M}\left(H_{1}\right)=0$. Более того, справедлив следующий вариант ${ }^{1)}$ теоремы Гельфанда-Манина-Шубина [9]:

\footnotetext{
1) При этом $\operatorname{grad}_{M}-$ аналог вариационной производной в теории уравнений в частных производных.
} 
ПрЕДЛОЖЕНИЕ 5. Пусть $a \in \mathcal{M}$. Тогда $a \sim P(C)$, əде $P$ - некоторый многочлен, если и только если $\operatorname{grad}_{M}(a)=0$.

Для примера проверим, что уравнение (2) записьвается в гамильтоновой форме (26) с гамильтонианом $H(M, C)=C \circ M^{2}$ и гамильтоновым оператором $\Theta=\operatorname{ad}_{M}$. Для этого достаточно показать, что $\operatorname{grad}_{M}(H(M, C))=M \circ C+C \circ M$. Действительно,

$$
H(M+\epsilon \delta M, C)=H(M, C)+\epsilon C \circ \delta M \circ M+\epsilon C \circ M \circ \delta M+O\left(\epsilon^{2}\right)
$$

и

$$
\begin{gathered}
\left.\frac{d}{d \epsilon} H(M+\epsilon \delta M, C)\right|_{\epsilon=0}=C \circ \delta M \circ M+C \circ M \circ \delta M \sim \\
\sim \delta M \circ(M \circ C+C \circ M)=\delta M \circ \operatorname{grad}_{M}(H(M, C)) .
\end{gathered}
$$

ОПРЕДЕЛЕНИЕ 6 . Назовем $\Theta \in \mathcal{O}$ гамильтоновым оператором, если скобка

$$
\{a, b\}=\operatorname{grad}_{M} a \circ \Theta\left(\operatorname{grad}_{M} b\right), \quad a, b \in \mathcal{M},
$$

удовлетворяет следуюшим условиям:

$$
\begin{gathered}
\{a, b\}+\{b, a\} \sim 0, \\
\{a,\{b, c\}\}+\{b,\{c, a\}\}+\{c,\{a, b\}\} \sim 0,
\end{gathered}
$$

для любых $a, b, c \in \mathcal{M}$.

С помощью принципа подстановки [7] можно показать, что условие (27) эквивалентно условию

$$
\Theta^{\star}=-\Theta
$$

а тождество Якоби (28) - соотношению (совпадающему с (7.11) в [7])

$$
Q_{2} \circ \Theta_{t_{3}}\left(Q_{1}\right)+Q_{3} \circ \Theta_{t_{1}}\left(Q_{2}\right)+Q_{1} \circ \Theta_{t_{2}}\left(Q_{3}\right) \sim 0
$$

для любых $Q_{i} \in \mathcal{M}$. Здесь $d / d t_{i}-$ дифференщирования в силу потоков

$$
\frac{d}{d t_{i}} M=\Theta\left(Q_{i}\right)
$$

Легко видеть, что $\widehat{\Theta}=\operatorname{ad}_{C^{k}}=L_{C^{k}}-R_{C^{k}}$ - гамильтонов оператор для любого $k$. Действительно, он кососимметричен и удовлетворяет условию (30), т.к.

$$
\frac{d}{d t_{i}} L_{C^{k}}=\frac{d}{d t_{i}} R_{C^{k}}=0
$$

Оператор $\Theta_{1}=\operatorname{ad}_{M}=L_{M}-R_{M}$ также удовлетворяет условиям (29), (30) и поэтому является гамильтоновым оператором на $\mathcal{M}$. 
Однородные гамильтоновы операторы $\widehat{\Theta}$ и $\Theta_{1}$ имеют соответственно веса $(k, 0)$ и $(0,1)$. Легко видеть, что для любого $\lambda \in \mathbb{R}$ оператор

$$
\Theta(\lambda)=\Theta_{1}+\lambda \widehat{\Theta}
$$

является гамильтоновым. Другими словами, операторы $\widehat{\Theta}, \Theta_{1}$ согласованны и образуют гамильтонову пару. Как обычно, гамильтонова пара генерируется простым сдвигом: если мы заменим $M$ на $M+\lambda C^{k}$ в $\Theta_{1}$, мы получим

$$
\operatorname{ad}_{M+\lambda C^{k}}=L_{M+\lambda C^{k}}-R_{M+\lambda C^{k}}=\Theta_{1}+\lambda \widehat{\Theta}
$$

Условия (29), (30) являются рабочим инструментом для отыскания гамильтоновых операторов на $\mathcal{M}$. Применим метод неопределенных коэффициентов и найдем все однородные гамильтоновы операторы малых весов. При этом условия (29) и (30) приведут соответственно к системе линейных и квадратичных алгебраических уравнений для коэффициентов оператора $\Theta$.

ПРЕДЛОЖЕНИЕ 6. С точностью до растяжения $M$ каждый однородный гамильтонов оператор веса $(0, m), m<8$, или $(1, m), \quad m<7$, совпадает с одним из следующих:

$$
\begin{aligned}
& \Theta_{0}=R_{C}-L_{C}, \\
& \Theta_{1}=R_{M}-L_{M}, \\
& \Theta_{2}=L_{C} R_{M}-L_{M} R_{C}, \\
& \Theta_{3}=L_{M}^{2} R_{M}-L_{M} R_{M}^{2}, \\
& \Theta_{4}=L_{M} L_{C} L_{M} R_{M}-L_{M} R_{M} R_{C} R_{M} .
\end{aligned}
$$

Сдвиги $C \rightarrow C+\lambda I d$ и $M \rightarrow M+\mu I d$ в $\Theta_{2}$ дают согласованную гамильтонову тройку $\Theta_{2}+\lambda \Theta_{1}+\mu \Theta_{0}$. Нетрудно проверить, что уравнение (2) и его симметрии гамильтоновы относительно всех трех операторов $\Theta_{0}, \Theta_{1}$ и $\Theta_{2}$.

Сдвиг $C \rightarrow C+\lambda I d$ в $\Theta_{4}(36)$ приводит к согласованной паре $\Theta_{4}+\lambda \Theta_{3}$. Если предположить, что элемент $M$ обратим, формальная замена переменных $\widetilde{M}=M^{-1}$ связывает операторы $\Theta_{1}$ и $\Theta_{2}$ соответственно с операторами $\Theta_{3}$ и $\Theta_{4}$.

4.3. Рекурсионные операторы. Рекурсионный оператор, если он известен, дает удобный способ для описания всей иерархии симметрий для уравнения (1) [7].

Назовем $\Lambda$ рекурсионным оператором для уравнения (1), если $\Lambda$ удовлетворяет соотношению

$$
\Lambda_{t}=F_{*} \Lambda-\Lambda F_{*}
$$

Если $\Lambda$ - рекурсионный оператор, то

$$
M_{\tau}=\Lambda(F)
$$

4 Теоретическая и математическая физика, т. 122, № 1, 2000 г. 
является симметрией уравнения (1). Действительно, $\left(M_{t}\right)_{\tau}=F_{*} \Lambda(F)$ и

$$
\left(M_{\tau}\right)_{t}=\Lambda_{t}(F)+\Lambda\left(F_{t}\right)=\left(F_{*} \Lambda-\Lambda F_{*}\right)(F)+\Lambda F_{*} F=F_{*} \Lambda(F) .
$$

Легко видеть, что для любых операторов $\Lambda_{1}$ и $\Lambda_{2}$, удовлетворяющих уравнению (37), линейная комбинация $\alpha \Lambda_{1}+\beta \Lambda_{2}$ и произведение $\Lambda_{1} \Lambda_{2}$ также удовлетворяют (37). Следовательно, любая степень $\Lambda_{i}^{n}$ удовлетворяет $(37)$, и поэтому для любого $j$ потоки $M_{\tau_{j}}=\Lambda^{j}(F)$ являются симметриями для (1).

Для того чтобы сделать определение рекурсионного оператора строгим, мы должны уточнить природу оператора $\Lambda$, т.е. описать класс, к которому принадлежит $\Lambda$.

В хорошо изученном случае уравнений в частных производных бо́льшая часть рекурсионных операторов является псевдодифференциальными (более точно отношениями дифференциальных операторов). Существование локального (т.е. дифференциального) рекурсионного оператора безошибочно указывает на $C$-интегрируемость уравнения [3].

Сначала мы определим локальньй рекурсионньй оператор как элемент из $\mathcal{O}$, удовлетворяющий операторному уравнению (37), а затем расширим определение на "нелокальный" случай.

Проверим, что $\Lambda=L_{M}$ является локальным рекурсионным оператором для уравнения (7). На самом деле, согласно (7)

$$
\frac{d}{d t} \Lambda=L_{M^{2} C}-L_{M C M}
$$

Производная Фреше от правой части (7) определяется соотношением

$$
F_{*}=R_{M C}+L_{M} R_{C}-R_{C M}-L_{M C},
$$

и, следовательно,

$$
\begin{aligned}
F_{*} \Lambda-\Lambda F_{*}= & \left(R_{M C}+L_{M} R_{C}-R_{C M}-L_{M C}\right) L_{M}- \\
& -L_{M}\left(R_{M C}+L_{M} R_{C}-R_{C M}-L_{M C}\right)=L_{M^{2} C}-L_{M C M} .
\end{aligned}
$$

Таким образом, $M_{\tau_{1, n+2}}=L_{M^{n}}\left(M^{2} C-M C M\right)=M^{n+2} C-M^{n+1} C M, n \in \mathbb{N}$, задает серию симметрий для уравнения (7). Однако это не весь набор симметрий (11) (см. предложение 2). Как мы увидим далее, оставшиеся симметрии могут быть получены с помошью другого, “нелокального” рекурсионного оператора.

Для гамильтонова уравнения (26) оператор $\Theta$ удовлетворяет операторному уравнению (см. формулу (7.38) в [7])

$$
\Theta_{t}=F_{*} \Theta+\Theta F_{*}^{\star} \text {. }
$$

Если (39) имеет еше одно решение $\Theta_{1}$, то (нелокальный) оператор

$$
\Lambda=\Theta_{1} \Theta^{-1}
$$


является рекурсионным для уравнения (26). А именно, как легко проверить, $\Lambda$ удовлетворяет соотношению (37), а поток

$$
M_{\tau}=\Lambda \Theta\left(\operatorname{grad}_{M}(H(M, C))\right)=\Theta_{1}\left(\operatorname{grad}_{M}(H(M, C))\right)
$$

задает симметрию для (26). Действие оператора (40) определено не на всей алгебре $\mathcal{M}$, а только на образе $\operatorname{Im}(\Theta) \subset \mathcal{M}$ оператора $\Theta$. Если $\Theta$ имеет ядро, то действие $\Lambda$ на $\operatorname{Im}(\Theta)$ определено неоднозначно и, для того чтобы добиться однозначности, требуются дополнительные условия (обычно связанные с однородностью симметрий). Фактически та же проблема возникает и в случае уравнений в частных производных, где, например, для уравнения Кортвега-де Фриза при задании рекурсионного оператора требуется корректно определить действие оператора $D_{x}^{-1}$.

Как уже отмечалось, уравнение (2) обладает гамильтоновыми операторами $\Theta_{0}=$ $L_{C}-R_{C}, \Theta_{1}=L_{M}-R_{M}=\operatorname{ad}_{M}$ и $\Theta_{2}=L_{M} R_{C}-L_{C} R_{M}$. Поэтому $\Lambda=\Theta_{2} \operatorname{ad}_{M}^{-1}-$ peкурсионный оператор для (2). Выберем $M^{k}, k=1,2, \ldots$, в качестве базиса ядра $\operatorname{ad}_{M}$. Применяя оператор $\Lambda$ к $F=M^{2} \circ C-C \circ M^{2}$, получим

$$
\begin{aligned}
G= & \Lambda(F)=\Theta_{2}(M \circ C+C \circ M)+\sum \alpha_{k} \Theta_{2}\left(M^{k}\right)= \\
= & M^{2} \circ C^{2}+M \circ C \circ M \circ C-C \circ M \circ C \circ M-C^{2} \circ M^{2}+ \\
& +\sum \alpha_{k}\left(M^{k+1} \circ C-C \circ M^{k+1}\right) .
\end{aligned}
$$

Мы видим, что элементы ядра $\operatorname{ad}_{M}$ порождают симметрии $S_{1, k}=M^{k} \circ C-C \circ M^{k} \in$ $\mathcal{M}_{1, k}$. Правая часть $F=S_{1,2}$ и операторы $\Theta_{1}, \Theta_{2}$ однородны, и, если мы потребуем, чтобы элемент $G=S_{2,2}=\Lambda(F)$ был также однородным, мы должны положить $\alpha_{k}=0$. Легко видеть, что $S_{2,2}$ принадлежит образу $\operatorname{ad}_{M}$, т.е.

$$
S_{2,2}=\operatorname{ad}_{M}\left(M \circ C^{2}+C \circ M \circ C+C^{2} \circ M\right),
$$

и поэтому выражение $S_{3,2}=\Lambda\left(S_{2,2}\right)=\Lambda^{2}(F)$ корректно определено и задает следующий член иерархии симметрий для уравнения (2) и т.д.

Генераторы симметрий $S_{n, m}, n, m \in \mathbb{N}$, для (2) могут быть получены рекурсивно:

$$
S_{n+1, m}=\Lambda S_{n, m}, \quad S_{1, m}=M^{m} \circ C-C \circ M^{m}
$$

исходя из симметрий веса $(1, m)$. Последние симметрии генерируются с помощю соответствуюшего выбора элементов из ядра $\operatorname{ad}_{M}$ при применении оператора $\Lambda$ к нулевой симметрии.

Однако сушествует более изящное объяснение существования симметрий вида $S_{1, m}$. Поскольку уравнение (2) тригамильтоново, мы имеем еще один рекурсионный оператор $\Lambda_{1}=\Theta_{2} \Theta_{0}^{-1}$. Легко видеть, что

$$
S_{n, m+1}=\Lambda_{1} S_{n, m}, \quad S_{n, 1}=M \circ C^{n}-C^{n} \circ M
$$


(здесь мы также фиксируем возможньй элемент ядра $\Theta_{0}$ условием однородности). Применяя оба оператора $\Lambda$ и $\Lambda_{1}$ к простейшей симметрии $S_{1,1}=[M, C]$, можно получить любой элемент “двойной” иерархии $S_{n, m}=\Lambda^{n-1} \Lambda_{1}^{m-1}\left(S_{1,1}\right)$.

Та же самая иерархия симметрий может быть получена, конечно, с помощью первых интегралов $I_{n, m}$ :

$$
S_{n, m}=\operatorname{ad}_{M}\left(\operatorname{grad}_{M}\left(I_{n, m}\right)\right)=\Theta_{2} \operatorname{grad}_{M}\left(I_{n-1, m}\right)
$$

Легко проверить, что $\Theta_{3}$ и $\Theta_{4}$, задаваемые формулами $(35),(36)$, являются гамильтоновыми операторами для уравнения (17). Поэтому оператор

$$
\begin{aligned}
\Lambda & =\Theta_{4} \Theta_{3}^{-1}=\left(L_{M} L_{C} L_{M} R_{M}-L_{M} R_{M} R_{C} R_{M}\right)\left(L_{M}^{2} R_{M}-L_{M} R_{M}^{2}\right)^{-1}= \\
& =\left(L_{M} L_{C}-R_{M} R_{C}\right) \operatorname{ad}_{M}^{-1}
\end{aligned}
$$

есть рекурсионный оператор для уравнения (17). Базисные элементы $M^{n}$ ядра $\operatorname{ad}_{M}$ приводят к симметриям $S_{1, n+1}=M \circ C \circ M^{n}-M^{n} \circ C \circ M$. Исходя из этих симметрий и используя рекурсионный оператор (42), мы можем легко построить симметрии $S_{n+1, m}=\Lambda S_{n, m}$ для уравнения (17).

Предположим, у нас есть основания считать, что данное уравнение (1) на $\mathcal{M}$ интегрируемо и мы хотим найти для него рекурсионный оператор. Локальный оператор $\Lambda \in \mathcal{O}$ достаточно малого порядка мы без труда найдем прямо из уравнения (37). Но, как правило, локальных рекурсионных операторов не существует. Уравнение (1) может не иметь двух (или даже одного) локальных гамильтоновых операторов. Некоторую подсказку на то, что можно предпринять в этом случае, дает формула (42). Последнее в этой цепочке равенств - отношение операторов $Q_{1}=L_{M} L_{C}-R_{M} R_{C}$ и $Q_{2}=\operatorname{ad}_{M}$. Ни один из них не является гамильтоновым для уравнения (17), но оба удовлетворяют одному и тому же операторному уравнению

$$
Q_{t}=F_{*} Q+Q P
$$

где $P=L_{M} L_{C} R_{M}-L_{M} R_{M} R_{C}$ - некоторый локальный оператор. Более того, оператор $Q_{3}=L_{M} R_{M} R_{C}-L_{M} L_{C} R_{M}$ также удовлетворяет уравнению (43). Нетрудно проверить, что $\hat{\Lambda}=Q_{3} Q_{2}^{-1}$ является еше одним рекурсионным оператором для уравнения (17), на первый взгляд никак не связанным с гамильтоновыми структурами.

Рассмотрим обшее уравнение (1). Простой выкладкой доказывается следующее утверждение.

ПРЕДЛОЖЕНИЕ 7. Для любого $P$ и любъх двух решений $Q_{1}, Q_{2}$ уравнения (43) оператор $\Lambda=Q_{1} Q_{2}^{-1}$ удовлетворяет уравнению (37).

Уравнение (43) является обобшением двух упомянутых выше операторных уравнений. А именно, если $P=-F_{*}$, то (43) совпадает с уравнением (37) для рекурсионного оператора, а если $P=F_{*}^{\star}$, то мы приходим к уравнению (43) для гамильтонова оператора (39). 
Ясно, что для однородного уравнения $F \in \mathcal{M}_{n, m}$ оператор $P$ должен принадлежать $\mathcal{O}_{n, m-1}$, и мы можем пытаться методом неопределенных коэффициентов найти из (43) сразу три локальных оператора $P, Q_{1}, Q_{2}$. Если из каких-либо соображений оператор $Q_{2}$ известен, то ситуация резко упрощается: подставляя $Q=Q_{2}$ в (43), находим $P$, а затем при известном $P$ ишем $Q_{1}$.

Уравнение (9) не является гамильтоновым, но тем не менее имеет иерархию симметрий. Легко проверить, что операторы $Q_{n}=L_{M} L_{C}^{n}, n=0,1, \ldots$, удовлетворяют (43) с $P=R_{M} R_{C}$. Поэтому $\Lambda=Q_{2} Q_{1}^{-1}=L_{M} L_{C}\left(L_{M}\right)^{-1}$ является нелокальным рекурсионным оператором для (9), порождающим иерархию симметрий

$$
S_{n, 2}=\Lambda^{n-1}(M \circ C \circ M)=M \circ C^{n} \circ M, \quad n=0,1,2, \ldots
$$

Аналогично уравнение (7), кроме локального оператора $\Lambda=L_{M}$, обладает нелокальным рекурсионным оператором $\Lambda_{1}=\operatorname{ad}_{M} R_{C} \operatorname{ad}_{M}^{-1}$. Этот оператор легко находится

из (43), если предположить, что $Q_{2}=\operatorname{ad}_{M}$. Рекурсионные операторы $\Lambda$ и $\Lambda_{1}$ совместно порождают иерархию симметрий

$$
S_{n, m}=\Lambda^{m-2} \Lambda_{1}^{n-1}([M, M \circ C])=\left[M^{m-1}, M \circ C^{n}\right], \quad n, m \in \mathbb{N},
$$

для (7).

Благодарности. Второй автор (В.В.С) благодарен организаторам конференции NEEDS-98 и Университету г. Лидса за гостеприимство. Работа была поддержана Российским фондом фундаментальных исследований (грант № 00294) и фондами EPSRC и LMS.

\section{Список литературы}

[1] V. V. Sokolov, A. B. Shabat. Soviet Scientific Reviews, Section C. 1984. V. 4. P. 221-280.

[2] А. В. Михайлов, А. Б. Шабат, Р. И. Ямилов. УМН. 1987. Т. 42. № 4. С. 3-53.

[3] A. V. Mikhailov, A. B. Shabat, V. V. Sokolov. The symmetry approach to classification of integrable equations. In: What is integrability? Ed. V.E. Zakharov. Berlin-N.Y.: Springer Verlag, 1991. P. 115-184.

[4] P. J. Olver, V. V. Sokolov. Commun. Math. Phys. 1998. V. 193. № 2. P. 245-268.

[5] P. J. Olver, V. V. Sokolov. Inverse Problems. 1998. V. 14. № 6. P. L5-L8.

[6] S. P. Balandin, V. V. Sokolov. Phys. Lett. A. 1998. V. 246. P. 267-272.

[7] П. Олвер. Приложения групп Ли к дифференциальным уравнениям. М.: Мир, 1989.

[8] В. В. Соколов, С. И. Свинолупов. Мат. заметки. 1993. Т. 53. № 2. С. 122-125.

[9] И. М. Гельфанд, Ю.И. Манин, М. А. Шубин. Функц. анализ и его прилож. 1976. Т. 10. № 4. C. $30-34$. 